\title{
ARTICLE 220 DE LA CONSTITUTION DU 18 FEVRIER 2006. Contribution au débat autour de son intangibilité et de sa « révisabilité »
}

\author{
Par Milambo NGALAMULUME GALANCE
}

\begin{abstract}
As part of this dissertation, it was a question of analyzing to what extent Article 220 of the Constitution of 18 February 2006 may be revised without this revision is bend the constitutional foundations of the Third Republic established by the original constituent. It is true that the inviolability of this provision is relative, because she first mission is to make intangibles other provisions. Far from our intention to say that this provision be revised to remove it all its legal force. Only a single revision-addition is possible to make Article 220 expressly intangible.
\end{abstract}

\section{Résumé}

Dans le cadre de cette dissertation, il a été question d'analyser dans quelle mesure l'article 220 de la Constitution du 18 février peut être révisé sans que cette révision porte entorse aux fondements constitutionnels de la troisième République établis par le constituant originaire. Il est certes vrai que l'intangibilité de cette disposition est relative, car elle a d'abord pour mission de rendre intouchable d'autres dispositions. Cela ne veut donc pas dire qu'elle est révisable en vue d'extirper la clause d'éternité. Seule une révision-ajout peut etre envisageable en vue de rendre l'article 220 expressément intangible.

\section{Introduction}

Il n'y a pas un débat qui a retenu l'attention, suscité d'intérêt et de polémiques tant par les chercheurs $^{2}$, les politiciens, que par le peuple congolais ces dernières années plus que celui relatif aux possibilités de modifier l'article 220 de la Constitution du 18 février 2006. A vrai dire, c'est la question liée à la « révisabilité » du nombre et de la durée des mandats du

1 Chercheur à l'Institut pour la Démocratie, la Gouvernance, la Paix et le Développement en Afrique. galance.milambo@gmail.com.

2 Boshab Evariste, Entre révision de la constitution et l'inanition de la Nation, Bruxelles, 2013; Mbata B. Mangu André, Monarchies présidentielles et révisions constitutionnelles : le syndrome du troisième mandat ou d'une présidence à vie dans les Etats membres de l'union africaine, RADG, vol.i., $\mathrm{n}^{\circ}$ 1, 2014; Akele Adau Pierre, Réponses pénales au discours du désordre ou au désordre du discours constitutionnel en République démocratique du Congo : la Cour constitutionnelle à l'épreuve, RADG, vol. $1, n^{\circ} 2$ et 3, 2014. 
Président de la République, du reste voulu intangible par le constituant originaire, qui est au cœur de cette discussion. Pourtant, c'est pour rompre avec les dérives du pouvoir ${ }^{3}$ que le constituant congolais de 2006 a institué les limites du nombre et de la durée de ces mandats, se référant ainsi à cette sagesse vénitienne selon laquelle, « la nature humaine est telle qu'on ne peut faire confiance à un homme qui a occupé longtemps, continûment un poste important de responsabilité »».

Est-ce pour autant affirmer « l'irrévisabilité » de la Constitution du 18 février 2006 ? Bien sûr que non! Une Constitution éternelle n'est possible que dans la cité de Dieu, tant que c'est une œuvre humaine, elle est soumise aux aléas de la vie politique, économique, sociale, voire au défi de générations qui passent. Etant la norme suprême, la Constitution accompagne les différentes mutations dans la légalité. À défaut, elle en subit les séquences.

Prenons donc garde! Tant il est vrai que la Constitution du 18 février n'est pérenne, tant il est aussi vrai que certaines dispositions voulues intangibles par le constituant, demeureront intouchables aussi longtemps que la Constitution tiendra. Ainsi, toute révision constitutionnelle qui se rapporte à l'article 220 tendant à le déverrouiller ${ }^{4}$, aurait un sens à peine voilé de faire souffrir le droit pour servir la politique. Ce serait donc une rébellion contre la Constitution dans la dimension d'un coup d'Etat constitutionnel, au point d'une transformation dangereuse de l'équilibre institutionnelle.

$\mathrm{Au}$ demeurant, il relève d'une évidence que les matières intangibles contenues dans l'article 220 constituent les fondamentales de la III ${ }^{\text {ème }}$ République, dans quelle mesure leur modification ne sera donc pas un passage en force vers la IV ème République? Aussi, comment cette disposition peut être révisée sans frauder à la Constitution? Cette intangibilité est-elle absolue ou relative?

Ces interrogations demandent un effort de démonstration qui passe par un cadre conceptuel (I), avant d'évoquer les fausses barbes soulevées par d'aucuns, pour légitimer une telle révision constitutionnelle (II), et les conséquences qui découleraient de cette révision (III). Pour finir, il sera impérieux d'analyser la place du juge constitutionnel devant une telle révision (IV). D'où la nécessité d'une approche critique pour ne pas nous scléroser dans un positivisme juridique excessif.

3 Il est établi depuis Montesquieu que le pouvoir absolu corrompt absolument. Ainsi, constate Olivier Duhame, « Le pouvoir trop prolongé corrompt plus profondément. L'homme de pouvoir tend à le conserver, l'homme au pouvoir veut s'y perpétuer. Autant le préserver de cette obsession, stimulante dans la langue marche vers le sommet, ravageuse dans sa trop longue occupation du poste suprême. Protégions le dirigeant contre lui-même. Les précédents récents. Si prestigieux soient-ils, l'attestent. Gonzalez, Kohl, Mitterrand, Thatcher, chacun d'entre eux aurait mieux gagné à s'arrêter plus tôt. Lire en ce sens Duhamel Olivier, le quinquennat, Paris, 2000, p.100.

4 Le référendum ne peut pas non plus résoudre le problème. Qu'il s'agisse du référendum d'en haut ou d'en bas déclenché respectivement parles pouvoirs publics et par l'initiative populaire. 


\section{A. Cadre conceptuel de l'intangibilité et de la révision constitutionnelle}

La maitrise des modalités de la « révisabilité » de l'article 220 passe inéluctablement par les débroussaillages du cadre conceptuel dont les éléments essentiels sont : la Constitution (I), la révision constitutionnelle (II), et les clauses d'intangibilité des dispositions constitutionnelle (III).

\section{Bref aperçu de la notion de Constitution}

D'une manière survolée, nous tenterons de définir la Constitution (1) avant de parler du pouvoir constituant (2).

\section{Constitution, quid?}

Il est dit que pour définir la Constitution, la référence à son objet, soit à sa matière, soit son contenu ${ }^{5}$ est inévitable. Est-il que même si on arrive à se référer à ceux-ci, l'équivoque entre Constitutions écrites et non écrites ou coutumières persiste pour autant ${ }^{6}$. L'institution d'un écrit traduit à coup sûr une nécessité de stabilité, même si, par ailleurs, le peuple préserve son droit pérenne de modifier sa Constitution. De ce point de vue, la Constitution peut être vue comme « l'instrument de la transparence du pouvoir, le point d'ancrage fixe, public et stable de la vie politique et juridique d'un pays $»$.

Par ailleurs, en fusionnant le critère formel et matériel de la Constitution, nous pouvons dire avec Marie-Anne Cohendet que la Constitution est «l'ensemble des règles considérées aux principes essentiels et aux normes de production de normes, et par conséquent situées au sommet de la hiérarchie des normes, et protégées par une procédure de révision plus complexe que celle des autres lois ${ }^{7} \gg$.

A tout bien pesé, la Constitution est la norme de référence, elle garantit l'équilibre de toutes les normes du droit interne puisqu'elle en est la source principale et la règle mère. Elle a un caractère suprême en ce qu'elle est le point culminant dans la hiérarchie des normes et aussi parce qu'elle est l'outil de la stabilité politique.

\section{Pouvoir constituant}

Sans entrer dans la forte controverse existant autour de cette notion, il y a lieu de retenir qu'il existe deux pouvoirs constituants: le pouvoir constituant originaire et le pouvoir constituant dérivé. Précisons pour demeurer exact que, ces deux pouvoirs n'ont pas la même souveraineté. Leur souveraineté varie selon que ce pouvoir est originaire ou dérivé.

5 Verpeaux Michel, La Constitution, Paris, 2008, p.104.

6 Boshab Evariste, 2, p.17.

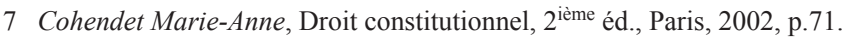


Le pouvoir est originaire lorsque effectivement, il se trouve être à la genèse d'un régime politique en élaborant une Constitution qui révèle être son assiette juridique. Dans ce cas, ce pouvoir constituant possède une souveraineté absolue. Par ailleurs, d'une manière principale, le pouvoir constituant est dérivé lorsqu'il est institué pour modifier la Constitution sans en renverser les fondements établis par le constituant originaire. Dans quel cas, la souveraineté de celui-ci est partielle.

Autrement, « le titulaire du pouvoir constituant originaire est le créateur d'une Constitution et celui du pouvoir constituant dérivé en est le réviseur. Le premier est un organe constituant qui tient sa compétence de lui-même, s'il n'est pas prévu par la Constitution en vigueur. Au regard de la Constitution à produire, il est « préconstitutionnel ». Le second, en revanche, est un organe constitué tenant de la Constitution sa compétence exclusive de révision de celle-ci $»^{8}$.

De la plume de Rousseau ${ }^{9}$ retenons donc que, « Réviser la Constitution est le travail d'un pouvoir institué qui a reçu cette compétence du pouvoir constituant originaire. Le premier est donc subordonné au second; son exercice n'est pas libre mais conditionné par les différentes règles de forme et de fond posées par le constituant originaire pour la révision de la Constitution; il peut dès lors être contrôlé ».

A titre illustratif, le peuple est le seul titulaire du pouvoir constituant originaire en République Démocratique du Congo. Il partage tout de même l'appartenance du pouvoir constituant dérivé avec le congrès.

Il est de l'essor du constituant dérivé d'aménager la procédure pour la révision de la Constitution en tenant compte : primo de l'organe investi de la compétence de sa révision, secundo des dispositions susceptibles de révision constitutionnelle, tertio les circonstances dans lesquelles la révision peut se réaliser. À des fois, il arrive qu'une Constitution brille par le mutisme sur la procédure de sa révision.

\section{Révision constitutionnelle}

L'occasion se prête pour dire ce qu'il faille entendre par révision constitutionnelle dans le cadre de ce travail (1), avant de déterminer l'organe détenant le pouvoir de révision en République Démocratique du Congo (2).

\section{Définition de la révision constitutionnelle}

En droit constitutionnel, les Constitutions sont sujettes aux modifications voire aux changements. L'imperfectibilité de l'œuvre humaine se vérifie aussi dans la Constitution. Car, aucune loi n'a la garantie de l'immuabilité aussi longtemps que la société dans laquelle elle est régie, ne l'est pas. L'évolution de la société, l'adaptabilité politique, les exigences cultu-

8 Sur d'autres détails concernant la distinction entre pouvoir constituant originaire et pouvoir constituant dérivé, voire Boshab Evariste, 3, p. 28-34.

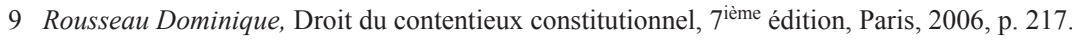


relles, aussi économiques sont entre autres, les raisons pour lesquelles une Constitution peut être modifiée.

Comme règle originaire et fondamentale, il revient de droit à la Constitution, de mettre l'État en position de mener une action durable au profit des citoyens ${ }^{10}$. L'essentiel est d'empêcher de réviser la Constitution " ni trop souvent, ni trop rapidement ${ }^{11}$ afin de protéger « le temps consolidé de la Constitution $»^{12}$. Une révision constitutionnelle n'est peut être appréciable et appréciée que dans la condition où elle améliore la situation préétablie, c'est donc un exercice pour combler les lacunes ou adapter la Constitution à la société; comme qui dirait : réviser la Constitution, c'est la remettre en chantier en vue de la perfectionner.

Il devient dès lors difficile d'accepter une quelconque révision totale de la Constitution sans frauder à la Constitution, sauf si la Constitution a expressément prévu sa révision partielle ou totale ${ }^{13}$.

En théorie, il existe une différence entre une révolution est une révision totale de la Constitution. Evariste Boshab signale que : « la différence entre la révolution et la modification totale réside dans le fait que la révolution construit du neuf en partant du néant; Elle n'est limitée par aucune règle, tandis que la révision totale débouche sur le même résultat quoiqu'elle ne bouleverse pas tout : Elle s'opère en respectant certaines règles édictées par la Constitution existante dont notamment les majorités à atteindre dans chaque chambre pour procéder à une révision totale ${ }^{14} »$.

Dans un autre registre, retenons que la révision de la Constitution s'articule autour de trois mouvements suivant : retoucher, extirper ou ajouter les dispositions constitutionnelles.

« Retoucher » une disposition constitutionnelle, c'est la modifier, la corriger partiellement en vue de l'amélioration et de l'adaptation aux exigences du temps. " Extirper » de son côté reviendrait à extraire, à arracher, à faire disparaitre d'une manière définitive une disposition qui existe dans la Constitution. Enfin « ajouter » une disposition constitutionnelle nous renvoie à la jointure des certaines dispositions afin de dire en plus de ce qui a été dit dans la Constitution pour une ample compréhension ${ }^{15}$.

10 Delperee Francis, Droit constitutionnel, Tome 1, Bruxelles, 1987, pp. 78-96.

11 Verdusen M. Stéphane, La procédure de révision de la Constitution: données comparatives, in Delperee Francis, et H. De croo, La procédure de révision de la Constitution, Bruxelles, 2003, p. 96.

12 Expression chère à Gianformaggio citée par Verdusen, 2, p.96.

13 Article 192 de la Constitution suisse dispose que : « la Constitution peut être révisée en tout temps totalement ou partiellement ». Il y a lieu de noter qu'en 1999, la Suisse a procédé à une révision totale de sa Constitution. Il y a eu substitution de la Constitution. Lire en ce sens Boshab Evariste, 4, pp.26-27; lire aussi dans ce sens l'article 195 de la Constitution helvétique qui dispose que : « la Constitution révisée totalement ou partiellement entre en vigueur dès que le peuple et les Cantons l'ont acceptée.

14 Boshab Evariste, 5, p. 39.

15 Notons que dans le cadre de cette dissertation, lorsqu'on parle du bien fondé de la révision de l'article 220, il va sans dire qu'on s'attarde sur le mouvement « ajouter » non sur « extirper » et « retoucher ». C'est seulement dans ce cas, que cette révision peut être légitime. 
Somme toute, pour défendre la stabilité constitutionnelle, chaque révision doit être justifiée. C'est le cas : des accords internationaux qui exigent la modification de la Constitution pour leur entrée en vigueur ${ }^{16}$, les normes transitoires concernant la continuité des institutions du moment que les nouvelles institutions sont mises en place ${ }^{17}$, les dispositions fixant des délais qui sont arrivés ou sur le point d'arriver à échéance...

\section{Détenteur du pouvoir de révision en République Démocratique du Congo}

L'initiative de la révision de la Constitution en RDC appartient concurremment au Président de la République, au Gouvernement après délibération en Conseil des ministres, à chacune des Chambres du Parlement à l'initiative de la moitié de ses membres, à une fraction du peuple congolais constituée de 100.000 personnes, s'exprimant par une pétition adressée à l'une des deux Chambres ${ }^{18}$.

Dans les limites de sa compétence, le Parlement peut contrôler l'initiative (projet ou proposition) ou d'une pétition qu'on lui sommet tendant à la révision constitutionnelle avant de la soumettre au vote du peuple soit au vote du Congrès ${ }^{19}$. A ce stade, il peut demander l'avis de la Cour constitutionnelle.

L'analyse de la conformité de la demande faite, deux voies s'ouvrent aux fins d'approbation de la révision constitutionnelle. Soit la voie référendaire, soit la voie du Congrès ${ }^{20}$.

Contrairement à ce qu'indique $B_{0 s h a b}{ }^{21}$, la voie référendaire est une voie de principe consacrée par le constituant originaire pour toute révision constitutionnelle et la voie de Congrès en est une exception. Pour arriver à une telle conclusion, il ne suffit pas de prendre le quatrième alinéa, mais d'analyser plutôt les alinéas 3 et 4 de l'article 218 de la Constitution $^{22}$. Car, l'alinéa 3 pose le principe et l'alinéa 4 pose l'exception introduite par « toutefois $^{23} \gg$.

16 Lire en ce sens l'article 216 de la Constitution du 18 février 2006.

17 Boshab Evariste, Les dispositions constitutionnelles transitoires relatives à la Cour constitutionnelle de la République Démocratique du Congo, Fédéralisme Régionalisme, Volume 7, n¹, 2007.

18 Lire en ce sens l'article 218 alinéa 1 de la Constitution du 18 février 2006; Cette initiative porte plusieurs dénominations selon leurs auteurs. Il s'agit d'une pétition si elle émane d'une portion du peuple congolais, d'un projet si elle est issue de l'Exécutif et d'une proposition, si elle provient d'une Chambre du Parlement.

19 Lire en ce sens l'article 218 al.2.

20 Sur la question de l'organe en même d'approuver la révision constitutionnelle nous pouvons lire Formery Simon-Louis, La Constitution commentée. Article par article, 16e éd., Paris, 2013/2014, ad art. 89, p. 169; Boshab Evariste, 6, pp. 319 et 322.

21 Boshab Evariste, 7, p. 322-323.

22 Lire en ce sens l'article 218 de la Constitution.

23 Yatala Nsomwe Ntambwe Constantin, La révision constitutionnelle et l'intangibilité de l'article 220 de la Constitution congolaise, http://www.droitcongolais.info/files/REVISION-220-CSTRDC-II.p df, (consulté le 12 décembre 2014). 
C'est avec raison que Frederic Rouvillois constate que « le peuple, titulaire normal du pouvoir constituant délégué, se trouve en pratique supplanté dans ce rôle par un organe constitué, le Congrès ${ }^{24}$ ». Il est claire d'admettre que cette constatation trouve sa raison d'être en ce que domine la conception formelle de la notion de pouvoir constituant. L'accessoire, le contingent, le subsidiaire deviennent ainsi la norme, le modèle et presque le principe de la révision de la loi fondamentale : la voie qui devait seulement servir aux réformettes est utilisé pour introduire des modifications essentielles ${ }^{25}$.

\section{Clauses d'éternité ou intangibilité}

1. Qu'est-ce que c'est qu'une clause d'éternité?

En vue de pallier les révisions intempestives de la Constitution, les dispositifs de sécurité doivent être pris nécessairement. Les dispositions intangibles sont donc des garde-fous placés pour pérenniser les valeurs fondamentales d'un système politique. Dans le but de combattre les abus du pouvoir dans le passé, ces dispositions protègent la Constitution des révisions à temps et à contretemps.

Il n'est strictement pas interdit de soulever le débat doctrinal sur la valeur juridique de l'intangibilité des dispositions constitutionnelles. Mais révolu est le temps de s'interroger sur l'importance et l'utilité de ces dispositions dans une Constitution rigide. Car, ces dispositions relèvent d'une certaine «morale juridique ${ }^{26}$ ».

D'une manière exhaustive, il existe trois arguments limitant le pouvoir de révision constitutionnelle, à savoir : l'argument de la « supraconstitutionnalité », l'argument des limites matérielles positives, et l'argument des limites intrinsèques au pouvoir de révision constitutionnelle.

S'agissant de l'argument sur la « supraconstitutionnalité », il faut noter qu'il existe certaines dispositions qui ne sont susceptibles d'aucune révision parce qu'elles expriment des normes de quelques manières supérieures aux autres normes constitutionnelles qui du reste, ne seraient que leurs dérivées. Les normes « supraconstitutionnelles » expriment le fondement du système constitutionnel. De ce fait, réviser une telle norme, c'est scier l'arbre sur lequel se fonde l'État.

L'argument des limites matérielles positives est le plus répandu. C'est d'abord dans la Constitution qu'on trouve les limites matérielles de la révision constitutionnelle. C'est-àdire, qu'il existe un certain nombre de dispositions constitutionnelles ne pouvant être révisé parce que le texte lui-même en interdit formellement.

24 Rouvillois fréderic, Droit constitutionnel. 2. La Ve République, Paris, 2004, p.95.

25 A titre illustratif, dès 1958 en France, le recours au congrès était manifeste que si la modification dont question est trop insignifiante pour intéresser directement le souverain : c'est pourquoi le Général De Gaulle a affirmé que « c'est bon pour les réformettes ».

26 Pour utiliser le terme de Yatala Nsomwe Ntambwe. 
L'argument des limites intrinsèques au pouvoir de révision constitutionnelle, n'est pas fréquemment exprimé entant que tel. Ce qui importe ici, c'est seule la compétence naturelle du pouvoir de révision qui fait de sorte que certaines dispositions constitutionnelles ne se révisent pas. Ce pouvoir est donc limité et le détenteur de ce pouvoir doit l'exercé dans les limites qui lui sont fixées.

Les deux premiers arguments se fondent sur la nature des dispositions remises en cause. Respectivement sur le contenu et sur leur caractère normatif de celles-ci. Pourtant, le troisième argument met un accent particulier sur la nature du pouvoir de révision lui-même. Il y a néanmoins une difficulté non moins négligeable. Ces arguments s'entrechoquent très souvent dans une même démonstration jusqu'à ce qu'ils deviennent difficilement repérables. Toutefois, les limites intrinsèques au pouvoir de révision sont souvent présentées comme structurantes pour le système constitutionnel et se rapprochent des normes « supraconstitutionnelles ». De même, les limites matérielles sont souvent interprétées de manière plus ou moins restrictive.

\section{Les clauses d'éternité à travers le monde}

Les dispositions intangibles ne sont pas une spécialité purement africaine comme le semble affirmer Boshab ${ }^{27}$. Elles ne sont pas non plus une nouveauté congolaise. Le droit comparé démontre qu'il en existe aussi sur d'autres cieux. En évitant de revivre les erreurs du passé ni de les transférer aux générations futures, certains États se sont vus obliger de verrouiller certaines dispositions.

A titre illustratif nous pouvons citer l'article 79 alinéa 3 de la Loi fondamentale de la République fédérale d'Allemagne du 23 mai $1949^{28}$, l'article 139 de la Constitution italienne ${ }^{29}$, l'article 89 de la Constitution française ${ }^{30}$, les articles 193 et 194 de la Constitution suisse $^{31}$, ne peut faire l'objet d'aucune révision, les articles 195, 196 et 197 de la Constitu-

27 Boshab Evariste, 8, pp. 117 et 120.

28 Cet article institue l'intangibilité : de principe démocratique, du fédéralisme, de la séparation des pouvoirs $(\ldots)$.

29 Il institue l'intangibilité de la forme républicaine.

30 Ici, aucune révision constitutionnelle ne peut porter atteinte à l'intégrité du territoire ou porter atteinte à la forme républicaine.

31 En suisse la révision de la Constitution, qu'elle soit partielle ou totale, ne doit pas porter atteinte aux règles impératives du droit international. 
tion Belge ${ }^{32}$, l'article 148 de la Constitution roumaine ${ }^{33}$, les articles 110, 2, 4, 5, 13,26 de la Constitution Grecque ${ }^{34}$, l'article 288 de la Constitution portugaise ${ }^{35}$, l'article 60 de la Constitution brésilienne de 1988 telle qu'amendée dans sa version de $1998^{36}$.

Les États africains ont aussi incorporé dans la plupart de leurs Constitutions des clauses d'intangibilité de révision. Nous citons : l'Algérie ${ }^{37}$, le Sénégal ${ }^{38}$, le Burkina Faso ${ }^{39}$, le Gabon $^{40} \ldots$

Il est sans nul doute vrai que l'article 220 de la Constitution du 18 février 2006 telle que modifiée parait plus prolixe en matière des clauses d'intangibilité à comparer aux autres États cités sus but, « les expériences connues par le passé, tel que le monopartisme, la compression des droits et libertés individuelles et collectives, tant politiques que syndicales, l'indépendance émoussée du pouvoir judiciaire, le simulacre du principe de la séparation des pouvoirs, la contingence intempestive des rapports entre le gouvernement central et les entités périphériques lors de nombreuses révisions et réformes constitutionnelles antérieures n'ont pas échappé à l'attention du Constituant du 18 février $2006^{41} »$.

32 Constitution telle qu'amendée et publiée au Moniteur belge du 29 décembre 2008, le pouvoir législatif fédéral peut modifier n'importe quelle disposition constitutionnelle qu'il indique. Toutefois la Constitution interdit la révision en temps de guerre ou lorsque les Chambres se trouvent empêchées de se réunir librement sur le territoire fédéral ou durant la régence il est interdit d'apporter des modifications à la constitution en ce qui concerne notamment les pouvoirs constitutionnels du Roi.

33 Il établit l'indépendance de la justice, le pluralisme politique, le caractère national, indépendant, unitaire et indivisible de l'Etat roumain, la forme républicaine de gouvernement, l'intégrité du territoire etc. sont à l'abri de toute révision constitutionnelle.

34 Cette disposition interdit le fait de porter atteinte au régime parlementaire, aux principes du respect et de la protection de la dignité humaine, aux principes de l'égalité, de la participation, de l'inviolabilité de la liberté, la liberté de conscience, la séparation des pouvoirs.

35 Il s'agit notamment du principe de l'indépendance nationale et de l'unité de l'Etat, les libertés fondamentales et garanties des citoyens, le suffrage universel direct et secret, l'autonomie politicoadministrative des archipels d'Açores et de Madère.

36 Au Brésil échappent à la révision constitutionnelle notamment le principe de la séparation des pouvoirs, les droits et les garanties individuelles.

37 Un aperçu général et à titre indicatif nous renseigne que la forme républicaine, l'islam comme religion d'État, les libertés fondamentales ainsi que le multipartisme ne peuvent faire l'objet d'aucune révision constitutionnelle en Algérie.

38 Au Sénégal seule la forme républicaine est protégée contre toute révision (art.103 in fine de la Constitution du 22 janvier 2001);.

39 Au Burkina Faso, aux termes de l'article 165 de la Constitution du 02 juin 1991 telle que révisée le 22 janvier 2002, ne sont susceptibles de révision constitutionnelle la forme et la nature républicaine de l'État, le multipartisme ainsi que l'intégrité territoriale.

40 Au Gabon, la Constitution révisée le 19 août 2003, en son article 117 met à l'écart des aléas de la révision la forme républicaine et le multipartisme.

41 Kazadi Mpiana Joseph, La révision constitutionnelle congolaise du 20 janvier 2011 : considérations critiques d'un citoyen (juriste). p. 12, http://www.droitcongolais.info (consulté le 15 décembre 2014). 


\section{B. Fausses barbes pour légitimer la révision de l'article 220 et leurs critiques juridiques}

Autant d'arguments fallacieux sont évoqués en vue de réviser l'article 220 de la Constitution du 18 février 2006, dans le sens d'extirper ou de retoucher sa teneur. Au nombre desquels, relevons que : une génération ne doit ni ne peut assujettir les générations futures (I), le principe de parallélisme de forme peut être à la base de la révision de cet article (II), l'article 220 est trop prolixe en matière d'intangibilité contrairement à d'autres Constitutions à travers le monde (III).

\section{Une génération ne peut en assujettir une autre}

L'argument le plus patent pour soutenir la révision de l'article 220 est que le souverain primaire ne peut pas lier définitivement les générations futures. Le peuple est dynamique et mutable autant les situations. Un texte constitutionnel ne doit pas lier de manière définitive les générations futures d'un État. Celles-ci doivent demeurer libres de rechercher à améliorer le système actuel. La souveraineté ne peut donc être une propriété exclusive d'une génération $^{42}$. Ceci dit, la révision de toutes les dispositions constitutionnelles même celles intangibles doit être faite dans le respect de conditions qu'impose chaque génération.

Certains arguments sont alimentés par la Constitution française du 24 juin 1793 qui indique que : « un peuple a toujours le droit de revoir, de reformer et de changer sa Constitution. Une génération ne peut assujettir à ses lois les générations futures ».

Juridiquement, il est difficile de légitimer une telle thèse. C'est une volonté manifeste de frauder à la Constitution sans se référer à la volonté initiale du peuple.

De la plume de Yatala nous pouvons noter que : « la critique de leur intangibilité et la proposition de leur « révisabilité » sous prétexte de leur prétendue inutilité et pour laisser libres les générations à venir relèvent des considérations philosophiques, politiques, voire idéologiques. Le juriste ne peut faire sienne cette critique et cette proposition sans trahir, quelque part la science juridique ${ }^{43} »$.

On ne peut certainement pas perdre de vue que toute loi est adoptée en principe pour régir l'avenir. Raison pour laquelle, elle est assortie d'un effet immédiat, excepté le fait qu'elle prévoit un effet rétroactif ou qu'elle soit favorable au prévenu à l'instar de la loi pénale. Quoique l'on dise ou que l'on fasse, toute loi disposant pour l'avenir, lie les générations futures aussi longtemps qu'elle ne connaitra pas d'abrogation. La Constitution ne s'écarte aucunement de cette logique, autant elle aussi une loi au sens large du terme.

Retenons encore qu'au regard du droit congolais, une génération n'est pas un sujet de droit, soutient Yatala : « ni un organe pouvant être auteur d'un texte normatif. C'est une catégorie sociologique utilisée en droit, faute, peut-être, d'arguments juridiques solides ».

42 Gauchet Marcel, La Révolution des pouvoirs, Paris, 1995, p. 280.

43 Yatala Nsomwe Ntambwe Constantin, 2, p.4. 
Le plus drôle ce qu'une génération compte 25 ans, depuis l'adoption de la Constitution du 18 février 2006 jusqu'en 2016 on compte à peine 10 ans. De quelle génération s'agit-il? De plus, les mêmes personnes qui ont assisté aux négociations ayant abouties à la rédaction de cette Constitution, ce sont les mêmes personnes qui peuvent probablement demander la révision pour ne pas lier les générations futures. Point n'est besoin d'avoir une thèse en droit constitutionnel pour comprendre qu'il y a anguille sous roche.

\section{Le parallélisme de forme}

Le principe de parallélisme des formes et des compétences veut que l'autorité qui a pris un acte soit la seule qui soit en même de l'abroger en vertu de la théorie de l'acte contraire. Puisque c'est par référendum que le peuple congolais a adopté la Constitution du 18 février 2006 et a voulu verrouiller certaines dispositions. Il va sans dire qu'il est le seul pouvant le déverrouiller.

Rousseau n'affirmait-il pas qu'un peuple « est toujours maître de changer ses lois, même les meilleures car s'il lui plaît de se faire mal à lui-même, qui est-ce qui en a le droit de l'en empêcher? » Etant souverain, le peuple est en droit de répondre à toutes les questions liées à sa condition aussi longtemps qu'il n'est pas limité au droit. De ce fait, le référendum peut être convoqué à tout moment.

A dire vrai, un tel argument est une manifestation flagrante de la volonté de violer la Constitution. Poser une telle question au peuple, c'est faire de lui un instrument de blanchiment des hérésies juridiques ${ }^{44}$. À croire que le peuple congolais qui s'est obligé à respecter à travers la Constitution, la clause d'intangibilité, mieux, le principe de limitation des mandats présidentiels semble être largement incapable de l'observer et que ce principe s'avère être au-dessus de ses forces et parait utopique quant à sa mise en pratique.

En plus, théoriquement, il est possible d'interroger le souverain primaire sur une question non réglée par la Constitution à travers la procédure référendaire. Mais pour toute autre question déjà éclairée par le souverain, au nom du principe de la souveraineté, elle doit s'appliquer à tous. Au regard du droit constitutionnel, l'initiative référendaire quant à l'article 220 est dénouée donc de tout fondement et de toute base juridique. Un tel acte arbitraire ne peut qu'être considéré nul et de nul effet.

La Constitution a prévu la procédure référendaire et les matières pouvant y être soumise. On ne peut pas du tout s'en passer afin d'organiser anarchiquement le référendum en vertu du principe de la souveraineté. Pourtant, le respect formel et matériel de la Constitution doit être le curseur de tout référendum. Si la procédure référendaire ne pose pas des problèmes, il n'en est pas vrai des matières. En République Démocratique du Congo, nous n'avons pas l'équivalent de l'article 11 de la Constitution française qui laisse une large lati- 
tude en matière référendaire ${ }^{45}$. À la place, nous avons des articles prévoyant le référendum facultatif, soit obligatoire ${ }^{46}$.

Le peuple congolais a pour défis essentiel la préservation des principes démocratiques contenus dans la Constitution de 2006 contre les aléas de la vie politique et les tentatives de révisions constitutionnelles multiples, non une machine de légitimation des hérésies.

Contrairement à d'autres normes constitutionnelles, l'article 220 ne peut être révisé dans le sens de retoucher ou d'extirper, même par référendum. En excluant cette disposition de la « révisabilité », le constituant pose donc une limite matérielle au référendum. De ce fait, une révision constitutionnelle par référendum touchant les matières considérées intangibles est de nul effet et sans fondement juridique.

Le déverrouillage de l'article 220 ne peut se faire que par le même pouvoir qui l'a verrouillé en vertu du principe de parallélisme de forme. Ici, point ne besoin de rappeler qu'il ne s'agit nullement de ce peuple réformateur d'un acte déjà fait. Mais plutôt d'un pouvoir constituant originaire fondant un nouveau régime. Somme toute, il ne s'agira pas de réviser une Constitution qui ne prévoit pas sa révision totale, mais plutôt de mettre sur pied un nouveau pacte social.

\section{La prolixité de principes intangibles}

D'aucuns affirment que les dispositions constitutionnelles intangibles ne sont que de simple des « barrières de papiers ${ }^{47}$ », carrément, le chapelet de bonnes intentions.

Pourtant, les clauses de limitation de révision constitutionnelle sont généralement une manière pour le constituant de veiller à ce que l'État ne revive pas les erreurs du passé. Sorti d'une dictature de 32 ans, le constituant ne pouvait que tirer des leçons en limitant définitivement le nombre de mandats présidentiels à deux tours pour garantir l'alternance et d'éviter l'accaparement du pouvoir par une seule personne ou par un groupe des personnes.

Il est non moins pertinent d'indiquer de surcroit que, la Constitution originaire expose « la philosophie du plus jamais ça ». Il va sans dire que c'est des expériences malheureuses

45 L'article 11 al. 2 de la Constitution française dispose: « Le président de la République, sur proposition du gouvernement pendant la durée des sessions ou sur proposition conjointe des deux assemblées, publiées au Journal officiel, peut soumettre au référendum tout projet de loi portant sur l'organisation des pouvoirs publics, sur des réformes relatives à la politique économique, sociale ou environnementale de la nation et aux services publics qui y concourent, ou tendant à autoriser la ratification d'un traité qui, sans être contraire à la Constitution, aurait des incidences sur le fonctionnement des institutions ».

46 Les matières obligatoirement référendaires sont: le transfert de la capitale dans un autre lieu du pays (art. 2 al. 3), la cession, l'échange et l'adjonction de territoire (art. 214 al. 2).

47 Droin N., Retour sur la loi constitutionnelle de 1884: contribution à une histoire de la limitation du pouvoir constituant dérivé, RFDC, vol. 80, 2009, pp. 735-736. 
du passé, que les constituants originaires africains des années 1990 a tiré les leçons ${ }^{48}$. Sans doute pour rencontrer les attentes des peuples. Le combat d'un régime tyrannique, despotique, autoritaire, voire une monarchie présidentielle, s'effectue entre autres par l'institutionnalisation des dispositions capables de protéger et de garantir : la vie politique assainie, l'accumulation des conditions d'éligibilité à la présidence, la limitation à deux du nombre de mandats présidentiels ${ }^{49}$, la prestation de serment du Président élu, l'obligation de déclaration par les gouvernants de leurs biens, l'interpellation des ministres, la définition de la haute trahison ${ }^{50}$, la dépolitisation de l'armée ${ }^{51}$ ou encore la désobéissance civile à l'oppression... 52

\section{Intangibilité de l'article 220 et conséquences de sa révision}

Il est en ne peut plus normal de s'interroger sur comment les institutions qui sont issues de la Constitution peuvent réviser une disposition qui se veut intangible (a), et ce, sans tomber de la fraude intentionnelle de la Constitution (b)?

\section{Article 220 : intangibilité absolue ou relative?}

Il est dit que le verrou posé par l'article 220 laisse une ouverture qui blanchirait la révision des matières voulues intangibles par le constituant originaire. Car dit-on que cette disposition verrou les autres dispositions sans pour autant se verrouiller elle-même. De ce point de vue, elle peut être modifiée sans toucher aux matières verrouillées.

Cet argument soulève des inquiétudes énormes. Quelle serait alors la valeur de cette disposition intangible étant donné que toutes les dispositions qu'elle veut intangible deviendraient révisables? L'intangibilité deviendrait dès lors sans fondement constitutionnel. Pour garantir l'intangibilité que l'article 220 institue, elle ne doit pas être modifiée, dans quel cas, elle ne poursuivrait plus son objectif et elle provoquerait la fragilisation de son intangibilité.

Il est sans nul doute vrai que, l'article 220 est une disposition relativement intangible. Car au départ, elle n'est pas une disposition intangible mais plutôt celle qui veut intangible

48 Voir, par exemple, Les actes de la conférence nationale (Cotonou, du 19 au 28 février 1990), Cotonou, ONEPI, 1994, Commission constitutionnelle de la République du Burundi, Rapport sur la démocratisation des institutions et de la vie politique au Burundi, Bujumbura, août 1991.

49 Loada A., La limitation du nombre de mandats présidentiels en Afrique francophone, Afrilex, $\mathrm{n}$ ${ }^{\circ} 03,2003$, pp. 139 et s.

50 Par exemple, selon l'article 96 la Constitution centrafricaine du 27 décembre 2004, « Sont notamment considérés comme crimes de haute trahison : la violation du serment; les homicides politiques; l'affairisme; toute action contraire aux intérêts supérieurs de la Nation ».

51 A. Cabanis et M.L. Martin, Armée et pouvoir dans les nouvelles constitutions d'Afrique francophone, RJPIC, ${ }^{\circ} 3$, septembre-décembre 1998, pp. 276 et s.

52 Bolle Stéphane, Des Constitutions «made in » Afrique, CERDRADI, mai 2005, pp. 27-30. 
d'autres dispositions ${ }^{53}$. Nous sommes emmené à penser que le fait pour le constituant de 2006, de ne pas avoir expressément indiqué l'intangibilité absolue de l'article 220 est une lacune survenue sans doute, à la suite du regard imitateur qu'il portait sur l'article 89 de la Constitution français de 1958, sans évidemment tenir compte ni de controverse doctrinale qui sont créées autour de lui, mais aussi sans tenir compte de la société congolaise.

Point n'est besoin d'ajouter qu'une disposition qui viendrait « intangibiliser » d'autres dispositions, doit être elle aussi à son tour intangible, à l'instar de l'article 197 de la Constitution Belge ${ }^{54}$. L'article 220 devait plutôt prévoir sa propre intangibilité juste pour ne pas laisser la possibilité, au second degré, de réviser les matières dont elle institue intangible. Cette révision-ajout instituant clairement son intangibilité protègerait les matières intangibles qu'elle contient.

L'article 220 garantit l'intangibilité absolue d'autres dispositions comme évoqué sus but. Pourtant elle-même est relativement intangible. Aussi longtemps qu'il ne protégera pas sa propre révision, les ennemis de l'État justifieront toujours leur tentative de réviser cet article en vue de pérenniser le pouvoir. Il semble logique à nos yeux de réviser cette disposition non pas pour extirper ou pour retoucher, mais plutôt ajouter expressis verbis le verrou de cette même disposition comme c'est le cas sous d'autres cieux.

Cet article ne pourra certainement pas être révisé pour modifier le contenu des dispositions intangibles, encore moins pour en diminuer le nombre. Il ne peut être touché que pour « s'intangibiliser » ou « intangibiliser » d'autres dispositions constitutionnelles. C'est la raison pour laquelle nous parlons de la relativité de son intangibilité 55 .

\section{Fraude à la Constitution}

La révision d'une loi se justifie par son amélioration et la Constitution n'échappe pas à cette logique. La Constitution du 18 février 2006 ne peut être révisée, que s'il existe de motifs d'intérêt public au-delà de toute suspicion, dans le respect de la procédure qu'elle prévoit et des limites qu'elle fixe, notamment à l'article 220. Une révision défiant cette logique et sur-

53 L'article 220 de la Constitution énonce sept interdictions de fond faites à la révision constitutionnelle, en prévoyant des matières intangibles. Il s'agit de la forme républicaine de l'État, du principe du suffrage universel (article 5 al. 4), de la forme représentative du Gouvernement (article 90 al. 3), du nombre et de la durée des mandats du Président de la République (article 70), de l'indépendance du pouvoir judiciaire (article 149), du pluralisme politique et syndical (article 6). En outre, la norme interdit formellement toute révision constitutionnelle ayant pour objet ou pour effet de réduire les droits et libertés de la personne (Titre II et article 61) ou de réduire les prérogatives des provinces et des entités territoriales décentralisées. Sur les sources matérielles (raisons historiques, idéologiques, philosophiques, politiques et sociologiques) de toutes ces dispositions intangibles.

54 (Texte coordonné du 17 février 1994) qui énumère les matières ne pouvant pas faire l'objet d'une révision et qui se protège aussi elle-même contre toute révision Voici le libellé de cet article : "Pendant une régence, aucun changement ne peut être apporté à la Constitution en ce qui concerne les pouvoirs constitutionnels du Roi et les articles 85 à 88, 91 à 95,106 et 197 de la Constitution. ».

55 Yatala Nsomwe Ntambwe Constantin, 3, p.4. 
tout remettant en cause les règles de conduite pour toute révision constitutionnelle est un moyen pour tailler la Constitution à petit feu, jusqu'à ce qu'elle atteindra le seuil des antivaleurs dont nous nous sommes habitués depuis toujours. Et les barrières juridiques placées pour ne pas permettre au premier venu de modifier la Constitution, tomberont au fur et à mesure qu'il y aura révision jusqu'au point de méconnaitre la Constitution initialement adoptée par le peuple.

Pour peu que l'on veuille bien se donner la peine d'analyser les conséquences qui découleraient d'une telle révision, le passage en force vers une quatrième République (1) et la violation de la souveraineté nationale appartenant au peuple (2) ont attiré notre attention.

\section{Passage en force vers la quatrième République}

Point ne besoin de rappeler que la Constitution du 18 février 2006 telle que révisée en ce jour, ne prévoit que sa révision partielle et non totale. S'incruster dans l'exercice de révision des dispositions intangibles reviendrait à une révision totale de la Constitution. Il s'agit d'un bricolage constitutionnel qui constituerait une métamorphose de régime.

Faire sauter les verrous de l'article 220 serait un passage en force et serait une manière de cautionner l'idée selon laquelle que les africains francophones noirs ne se sont pas entièrement séparé de l'état de nature dans lequel le plus fort règne.

Comment pouvons-nous modifier l'article 220 qui constitue la charnière, l'axe central, le piédestal, l'armature de l'architecture constitutionnelle congolaise sans détruire par ce fait, l'édifice constitutionnel construit au prix des supplices incalculables?

Il n'y a donc pas possibilité de faire référence à la pratique suisse pour légitimer une quelconque révision totale de la Constitution, parce que la Constitution fédérale prévoit sa révision totale qui est l'œuvre du peuple et des cantons. Cette Constitution ne prévoit pas de limites matérielles autonomes pour sa propre révision. Celle-ci peut être totale ou partielle, ce qui compte le plus, c'est la légitimité de l'exercice. Pour que la révision soit valable, elle doit atteindre la majorité du peuple et des cantons.

Quand on lit attentivement Auer, Malinverni et Hottelier, l'éclaircissement et la justification qu'on peut donner à l'absence de limites matérielles de la révision de la Constitution Suisse est que « la légitimité du constituant dérivé, à savoir du peuple et des cantons, est jugée supérieure à celle non seulement de tous les organes de l'État, mais aussi du constituant originaire ", laquelle légitimité résulte, "d'une part, du caractère démocratique du processus de révision constitutionnelle et, d'autre part, du fait que les cantons, qui participent à ce processus, ont préexisté à la Confédération et ne doivent donc pas leur existence à la Constitution de 1948. On ne voit donc pas quel organe de l'État, au nom de quel principe, pourrait valablement empêcher le constituant souverain de modifier les bases mêmes du régime politique et constitutionnel de la Suisse ${ }^{56} »$.

56 À noter que l'unique limite matérielle à la « révisabilité » de la Constitution helvétique est hétéronome. C'est le jus cogens. En effet, d'après les articles 193 al. 4 et 194 al. 2 de la Constitution fédérale, les révisions de la Constitution doivent respecter les règles impératives du droit interna- 
Il ne faudra nullement prétexter une révision constitutionnelle alors que c'est le changement de la Constitution qui est visé, au risque de faire de cette Constitution un colosse au pied d'argile pouvant être renversé au gré de tout vent de doctrine.

En modifiant cette disposition, on prendra donc le risque d'altérer le compromis politique historique de Sun City, et par conséquent, la cohésion nationale et la paix sociale risque d'être aussi menacé.

Il faut être amnésique pour oublier que la révision de 2011 a laissé des séquelles que le peuple congolais gère jusqu'à présent. À titre de rappel, elle a supprimé l'élection du Président de la République à deux tours ${ }^{57}$ et les Parquets du pouvoir judiciaire en RDC ${ }^{58}$. Contre toute attente légitime, cette révision avait accordé aussi au Président de la République des pouvoirs additionnels, notamment de dissoudre une Assemblée provinciale et de relever un Gouverneur de province de ses fonctions ${ }^{59}$.

2. Violation du principe « supraconstitutionnel» de la souveraineté nationale

Le syllogisme est tel que : la souveraineté appartient au peuple, et la Constitution est un acte de la souveraineté du peuple. Or, la révision de l'article 220 est une violation à la Constitution. Donc, l'inconstitutionnalité de cette révision est une atteinte à la souveraineté nationale qui appartient au peuple.

Le déverrouillage de l'article 220 nous pousse à affirmer avec l'ensemble de l'opinion publique que c'est un coup de force constitutionnelle, une violation du principe de la souveraineté nationale qui appartient au peuple. Pour la doctrine de la souveraineté nationale ${ }^{60}$, la souveraineté réside dans la Nation, être collectif et abstrait considéré comme un tout indivisible, distinct des individus qui la composent. La Déclaration française des Droits de l'Homme et du Citoyen du 26 août 1789 s'est orientée dans ce sens, puisqu'elle énonce : Le principe de « toute souveraineté réside essentiellement dans la Nation ». " Nul corps, nulle autorité ne peut exercer d'autorité qui n'en émane expressément ». Il en est de même du constituant français de 1791 pour qui « la souveraineté est une, indivisible, inaliénable. Elle appartient à la Nation; aucune section du peuple, ni aucun individu ne peut s'en attribuer l'exercice ».

tional. Il faut préciser que ces dispositions, combinées avec les articles 139 al. 2 et 139 alinéa 2 de la Constitution fédérale, ont codifié une pratique inaugurée par l'Assemblée fédérale en 1995.

57 Art. 71 de la Const.

58 Article 149 de la Const.

59 Articles 197 et 198 de la Const.

60 La doctrine de la souveraineté nationale est inventée par les Monarchomaques à la Renaissance, puis préconisée par Montesquieu et par Emmanuel Joseph Abbé Sieyes. 
Il sied de retenir pour mémoire, qu'il existe certains principes supérieurs à la Constitution $^{61}$ et inhérents au système politique. Ces principes servent donc de curseur au pouvoir constituant originaire, et lient au même moment le constituant dérivé.

De ce fait, il y a lieu de relativiser la portée illimitée du pouvoir constituant originaire ${ }^{62}$. Car, son pouvoir est encadré par certains principes qu'on ne peut y déroger ${ }^{63}$. L'origine de ces principes sont diverses. Ils peuvent provenir du droit international telle que le jus cogens ou encore les principes établissant l'ordre public international que le constituant ne peut pas ne pas connaitre. Ces principes peuvent provenir également de la Constitution matérielle.

La souveraineté nationale fait partie de cette catégorie de principe « supraconstitutionnel » que le pouvoir réformateur ne peut pas y toucher. En France, la question de la «supraconstitutionnalité » du principe de la souveraineté nationale a été posée lors du débat sur la ratification du Traité de Maastricht. On a soutenu que le Traité de Maastricht remettait en cause le principe de la souveraineté nationale. Or, selon les défenseurs de cette idée, ce principe est de valeur "supraconstitutionnelle », et par conséquent il s'impose même à l'exercice du pouvoir de révision constitutionnelle.

Selon Léo Hamon, « au-dessus même des dispositions constitutionnelles écrites, il y a une sorte de super constitutionnalité à laquelle ni les assemblées, ni les majorités passagères de citoyens ne peuvent porter atteinte, parce que ces dispositions s'imposent à la nation (...) On parle aujourd'hui de droits fondamentaux de l'homme et l'on admet qu'une révision constitutionnelle n'est légitime que si elles respectent ces droits. Et le principe de la souveraineté nationale n'est pas moins impérieux que le principe des droits de l'homme ${ }^{64}$. L'auteur ajoute que, « quand on dit qu'un acte n'est valable que parce qu'il ne porte pas atteinte à la souveraineté nationale, cela veut dire que s'il y portait atteinte il ne serait pas valable $»^{65}$.

61 Genevois Bruno, Les limites d'ordre juridique à l'intervention du pouvoir constituant, RFDA, vol. 14, septembre-octobre 1998, pp. 912-913.

62 L'identité de celui qui procède à l'écriture du texte constitutionnel et le souverain primaire n'est qu'apparente et ne reflète pas la réalité comme nous éclaire Cayla Olivier, L'obscure théorie du pouvoir constituant originaire ou l'illusion d'une identité souveraine inaltérable in D. De Bechillon, V. Champeil-Desplats, P. Brunet, et E. Millard, (dir), L'architecture du droit. Mélanges en l'honneur de Michel Troper, Paris, 2006, pp.249-265.

63 C'est dans cette optique que s'était prononcée la Cour constitutionnelle italienne dans son arrêt n `1146 de 1988. Il a d'abord dégagé des principes « qui bien que n'étant pas expressément mentionnés parmi ceux qui ne peuvent faire l'objet d'une procédure de révision constitutionnelle appartiennent à l'essence des valeurs suprêmes sur lesquelles se fonde la Constitution italienne (...). En admettant sa compétence pour se prononcer sur la conformité des lois de révision constitutionnelle à l'égard des principes suprêmes de l'ordonnancement constitutionnel, elle justifie cette position en déclarant que s'il n'en était pas ainsi..., on parviendrait à l'absurdité de considérer le système de garantie juridictionnelle de la Constitution comme défectueux et non effectif justement en relation avec ses normes dont la valeur est la plus élevée.

64 Hamon Lambert, La souveraineté nationale, la Constitution et les négociations 'européennes' en cours, 43 e Cahier, Chronique - LV, Recueil Dalloz Sirey, 1991, p. 303.

65 Hamon Lambert Idem, p.303. 
A son tour, Olivier Beaud dote la souveraineté nationale d'un statut de « supraconstitutionnalité », d'un principe de l'inaliénabilité du pouvoir constituant ${ }^{66}$, comme la forme moderne de l'inaliénabilité de la souveraineté ${ }^{67}$. Pour lui, le pouvoir de révision constitutionnelle est limité par ce principe. Ainsi, les dispositions de la Constitution touchant à la souveraineté du peuple ne peuvent être révisées que par le pouvoir constituant originaire, non pas par le pouvoir de révision ${ }^{68}$. Car si ces dispositions peuvent être révisées par le pouvoir de révision, « la nature systématique et fondatrice de la souveraineté devait donc s'effacer devant n'importe quel article voté par le législateur constitutionnel $»^{69}$. Ainsi selon Olivier Beaud, la non-limitation matérielle du pouvoir de révision « rend la souveraineté de l'Etat et du peuple à la merci d'un simple vote de parlementaires réunis en Congrès. Ce qui revient à affirmer que le Parlement réuni en formation de révision redevient le souverain ${ }^{70}$. Pourtant, le législateur constitutionnel n'est pas souverain ${ }^{71}$. En vertu du principe de l'inaliénabilité du pouvoir constituant, seul le peuple «peut remettre en cause la souveraineté $»^{72}$.

Pour sa part, Léo Hamon pense qu'il y a dans la Constitution « des principes fondamentaux sur lesquelles on ne peut pas revenir ». Et selon lui, la souveraineté nationale entre dans la catégorie de tels principes. Le professeur Hamon affirme qu'« un Parlement supprimant la souveraineté nationale, ou la mutilant, agirait aussi gravement qu'un Parlement prétendant établir des distinctions de droit entre les français selon leur religion, leur race, leur classe $»$.

Somme toute, l'affirmation selon laquelle le pouvoir de révision ne peut pas réviser les dispositions de la Constitution touchant à la souveraineté nationale du peuple ne résulte pas seulement de la Constitution du 18 février 2006 mais aussi d'un certain principe de " l'inaliénabilité du pouvoir constituant originaire » dont la source textuelle reste inconnue. Voilà pourquoi Olivier Beaud propose avec raison une hiérarchisation entre les dispositions de la Constitution en fonction du fait qu'elles touchent ou non à la souveraineté du peuple. Par conséquent il attribue une place supérieure aux dispositions de la Constitution touchant à la souveraineté du peuple.

\section{Contrôle de constitutionnalité de la loi constitutionnelle révisant l'article 220}

Prendre l'initiative de vouloir déverrouiller l'article 220 de la Constitution, est un acte non conforme à cette dernière et doit être soumise au contrôle de constitutionnalité ainsi pour

66 Beaud Olivier, La puissance de l'Etat, Paris, 1994, p.325.

67 Beaud Olivier, 2, p. 324.

68 Beaud Olivier, 3, p.1059.

69 Beaud Olivie, 4, p.476.

70 Beaud Olivier, La souveraineté de l'Etat, le pouvoir constituant et le Traité de Maastricht : remarques sur la méconnaissance de la limitation de la révision constitutionnelle, Revue française de droit administratif, 1993, p.1059.

71 Beaud Olivier, 5, pp.323-325.

72 Beaud Olivier, 6, p.476. 
être déclaré nul de plein droit ${ }^{73}$. Il est vrai que le souverain primaire ne prend pas part à l'initiative de la révision constitutionnelle. Cette tâche est réservée soit à ses représentants, soit à une portion du peuple, mais le juge constitutionnel doit cependant jouer le rôle de dernier rempart contre les violations de normes constitutionnelles. Par ce fait, deux types de contrôle s'offrent à lui : le contrôle a priori et le contrôle a fortiori.

Le contrôle a priori est ce type de contrôle qui intervient avant la promulgation de la loi, il est obligatoire en pratique pour les lois organiques, est à la fois préventif et abstrait. En effet, la loi votée n'est pas exécutoire et ne peut produire des effets juridiques qu'après sa promulgation ${ }^{74}$. De l'avis de Michel Fromont, puisque ce genre de contrôle n'a pas l'objectif de trancher un litige concret, il s'évertue par le contrôle à régler une querelle juridique entre les acteurs politiques, à savoir : le Président de la République et les députés. Ce contrôle permet somme toute, à discipliner et à encadrer les acteurs politiques tout en faisant du juge constitutionnel à la fois un arbitre du jeu politique et un précepteur des gouvernants ${ }^{75}$. Loin d'être instrumentalisé par le pouvoir comme tel est souvent le cas en Afrique, le juge constitutionnel s'impose par ce contrôle comme un acteur décisif du respect des principes démocratiques.

Le contrôle a posteriori par contre, est ce type de contrôle qui est mis en œuvre après la promulgation de la loi. Ce contrôle revêt deux aspects : il s'effectue soit par voie directe, on peut aussi parler de l'action populaire ayant un caractère objectif et abstrait; soit par l'exception d'inconstitutionnalité qui relève du contrôle subjectif et concret. Notons cependant que, l'action directe est très proche de l'amparo ${ }^{76}$. Dans la plupart d'Etats, à l'instar du Bénin, dans l'action directe, tout citoyen est habilité à saisir le juge constitutionnel de tout acte contraire à la Constitution mais susceptible de produire des effets juridiques.

\section{Le contrôle a priori de la révision de l'article 220}

De prime à bord, il est important de signaler que le contrôle de constitutionnalité de la loi a pour essentiel objectif de garantir la suprématie de la norme suprême. Le fondement de ce contrôle reste bien évidemment que le peuple exprime, directement et expressément, sa volonté dans la Constitution. Ce faisant, la volonté du peuple doit nécessairement être supérieure à la volonté de ses représentants qui s'expriment tout naturellement par la loi. C'est sur cette base que la loi portant révision de l'article 220 de la Constitution doit être contrôlée.

Pour sa part, le contrôle de constitutionnalité de la loi constitutionnelle consiste en fait, à l'analyser pour déterminer si l'autorité qui en a pris l'initiative est habilitée à le faire de

73 Art. 168, al. 2.

74 Holo Théodore, Emergence de la justice constitutionnelle, Pouvoir, pp. 77-90, www.afrilex.com (consulté le 15 juin 2014).

75 Holo Théodore, 2, p.78.s.

76 Recours en protection d'un droit constitutionnel protégé contre tout acte public, loi, acte administratif, jugement. 
par la Constitution, si le quorum indiqué par la Constitution a été atteint lors de son vote par le Parlement, si elle n'a pas révisé les normes qui de par la Constitution ne peuvent faire l'objet d'une révision. C'est une manière de mettre en évidence les aspects du régime d'exercice du pouvoir constituant dérivé.

Ce faisant, quelle pourrait être la procédure à suivre pour faire invalider la révision constitutionnelle de l'article 220 violant la Constitution?

Aucune disposition constitutionnelle ni légale ${ }^{77}$ évoque expressément le contrôle de constitutionnalité d'une loi constitutionnelle. La Constitution se limite à placer des barrières en ce qui est de l'objet, de la période et la procédure de la révision constitutionnelle. Mais il s'avère utile de s'interroger sur le rôle du juge constitutionnel dans l'éventualité de la violation soit de l'objet, de la période ou de la procédure de laquelle révision dans le strict respect du pouvoir constituant.

D'emblée, on peut soutenir que la souveraineté du pouvoir constituant écarte la Cour constitutionnelle du contrôle de constitutionnalité de la loi constitutionnelle, de surcroit qu'il n'y a pas une autorisation expresse. Cette réflexion semble être d'une fertilité débordante. Cette vue ne constitue qu'une simple fiction, apparemment inspirée mais en réalité non.

Pousser par une telle conception, le professeur Ismaila Madior Fall ${ }^{78}$ pense que : « Dans l'Etat de droit, tout contentieux doit trouver une solution devant le juge, ne serait-ce que par la voie d'une interprétation audacieuse, mais logiquement défendable de la Charte suprême. Le juge constitutionnel, mieux que tout autre, est en effet prédisposé à donner du sens à des textes souvent très généraux. En l'absence de dispositions constitutionnelles ou législatives pertinentes, seul le juge de la Constitution est capable d'y obvier ${ }^{79}$.

Dans la même logique, Stéphane Bolle ${ }^{80}$, Renoux et Villiers ${ }^{81}$ estiment que « les juges, constitutionnels mais aussi administratifs, sont les producteurs d'une «Constitution bis » ... plus riche, plus complète et plus dense que la Constitution formelle ».

Nous restons fondamentalement convaincu que toute loi, même constitutionnelle, votée en violation de la Constitution reste susceptible de pourvoi en inconstitutionnalité. Si les États comme l'Allemagne et l'Italie qui ont connu respectivement les expériences du nazisme et du fascisme sont allés jusqu'à non seulement, instituer des dispositions immuables et intangibles, mais aussi à invalider des lois constitutionnelles, Nous pensons que, la RDC doit instituer l'obligation de conformité par le juge constitutionnel, de la volonté du législateur constitutionnel à l'expression référendaire originaire.

77 Il est fait allusion à la loi organique portant organisation et fonctionnement de la Cour constitutionnelle.

78 Fall Ismailla Madior, Une démocratie « ancienne » en mal de réforme : Rapport sur l'état de la démocratie et de la participation politique au Sénégal, Johannesburg, 2012, p.47.

79 Fall Ismailla Madior, 2, p.47.

80 Bolle Stéphane, 2, p. 14.

81 Renoux Thierry-Serge et M. Villiers, Code constitutionnel, édition 2005, Paris, Litec, p. 505. 
Par ailleurs, de l'interprétation de la Cour Constitutionnelle, doit être dégagé une analyse démocratique en même de « donner une extension que n'impliquait pas nécessairement la seule lecture ». Nous pouvons parler de la bonification de la Constitution, car, " c'est souvent la volonté de concrétiser un ordre fondamental en totale rupture avec l'ancien régime et de prévenir tout contournement de cet ordre qui transparaît d'un ajout jurisprudentiel $^{82} \gg$.

Ce faisant, seule la Constitution formelle ne suffit pas pour soumettre une loi constitutionnelle de cette ampleur à un contrôle de constitutionnalité, sa combinaison avec la Constitution matérielle est impérieuse.

La portée du recours aux principes non écrits sur la rigidité constitutionnelle doit préoccuper autant. Cette valorisation de principes vient contrer les latitudes du pouvoir constituant dérivé devant qui, elle vient dresser une nouvelle épreuve après celle de la satisfaction des conditions de procédures ${ }^{83}$ et des limitations matérielles ${ }^{84}$ du pouvoir de réviser. On y trouve un nouvel aspect de la rigidité constitutionnelle qui ne s'exprime plus seulement dans les limites formellement établies par la Constitution mais qui plonge dans le fondamentalisme constitutionnel pour rechercher et faire imposer les options fondamentales, idéologiques et politiques qui ont présidé à l'avènement de la Constitution, de sorte à les placer côte à côte avec le contenu formel de la Constitution. De ce fait, toute révision constitutionnelle doit satisfaire, et la Constitution matérielle, et la Constitution formelle. Faute de quoi, elle doit être considérée d'inconstitutionnel.

\section{Exception d'inconstitutionnalité}

Une frange doctrinale martèle sur le caractère général de l'article 162 alinéas 1 et 3 de la Constitution «prévoyant la possibilité de soulever une exception d'inconstitutionnalité contre n'importe quel acte permet également à la Cour constitutionnelle, saisie dans le cadre d'un litige pendant devant une juridiction, d'assurer le respect des règles de révision constitutionnelle $»^{85}$.

Qu'adviendrait d'une disposition constitutionnelle soulevée en exception d'inconstitutionnalité, la demande serait-elle déclarée irrecevable pour incompétence de la Cour? Cependant, qui peut avoir la qualité pour saisir la Cour constitutionnelle? À cet effet, est-ce

82 Cette volonté est en général tacite. Par exception, le Haut conseil de la République du Bénin siégeant en qualité de Cour constitutionnelle, dans sa Décision n¹4 DC du 16 février 1993, Rec. p. 55, a estimé que, s'agissant de la Haute autorité de l'audiovisuel et de la communication, «l'esprit qui a prévalu à l'installation d'une telle institution résulte indubitablement de la confiscation de la liberté de presse par l'ancien régime et ce pendant plusieurs années ». Lire aussi constitution made africa.

83 Mbata B. Mangu André, 2, p.50.

84 Mbata B. Mangu André, 3, p. 50.

85 Banneux Nicolas, Boshab Evariste, Bossuyt Marc, Kabamba Bob et Verjans Pierre, http://popups.u lg.ac.be/federalisme/document.php?id=209, consulté le 29 janvier 2010 et la note 116. 
seulement les institutions habilitées à initier le référendum ou tout citoyen qui s'estime lésée virtuellement par la révision envisagée?

Les articles 160 al.1 et 162 al. 2 semblent être clairs de l'avis de Yatala, car dit-il que le contrôle la constitutionnalité des actes législatifs et des actes réglementaires ayant force de loi entre dans la compétence de la $\operatorname{Cour}^{86}$. De ce fait poursuit-il, en dehors des institutions habilitées à saisir la Cour, tout citoyen pourrait attaquer une révision constitutionnelle pour inconstitutionnalité.

Cette vue nous parait irréelle. On ne peut guère s'appuyer sur les articles sus cités pour légitimer l'exception d'inconstitutionnalité soulevée par tout citoyen devant la Cour constitutionnelle. L'exception d'inconstitutionnalité est soulève après la promulgation d'un acte législatif ou réglementaire et non avant. Or, toute loi constitutionnelle après promulgation fait corps avec la Constitution. Ce contrôle ne sera plus un contrôle de constitutionnalité d'autant plus qu'on ne contrôlera pas la constitutionnalité de ces actes mais plutôt la constitutionnalité de la Constitution. A notre avis, c'est seules les institutions ayant reçue attribution de la Constitution.

\section{Conclusion}

De l'accession à la souveraineté internationale vallonnée, aux différentes périodes de transition évoluant en dents de scie, en passant par la première République instable, à la deuxième République dictatoriale, aux guerres causant plusieurs millions de morts(...), la RDC s'est frayé un chemin tout autre en adoptant par référendum un nouveau pacte politique et social.

La sauvegarde du pacte républicain notamment par le strict respect de la Constitution principalement dans ses dispositions voulues intangibles par le constituant : la forme républicaine de l'État, le principe du suffrage universel, la forme représentative du gouvernement, le nombre et la durée du mandat du Président de la République, l'indépendance du Pouvoir judiciaire, le pluralisme politique et syndical, la non-réduction des droits et libertés de la personne, la non-réduction des prérogatives des provinces et des entités territoriales décentralisées.

Systématiquement, il est envisageable de parler de la protection des dispositions constitutionnelles intangibles, du reste sans angélisme ni pyrrhonisme. Au demeurant, la stabilité de l'architecture constitutionnelle se trouvera inexorablement compromises avec le déverrouillage de l'article 220.

Pour ceux-là qui font de l'alternance l'essence même de leur démocratie, la notion du mandat présidentiel, doit en être la pierre angulaire. Ce faisant, cette notion du mandat s'impose non seulement comme norme constitutionnelle d'autant qu'elle est précisée dans le texte constitutionnel, mais aussi est surtout entant que principe contraignant lié au consti-

86 Sur les notions d'actes législatifs comprenant les lois et les actes ayant force de loi, voir Vundwawe te Pemako Félix, p. 217ss; Arrêt Kapuku Ngoyi Trésor, de la CSJ, n RCONST. 51 / TSR du 31 juillet 2007; Arrêt RA. 320 de la CSJ du 21 août 1996. 
tutionnalisme démocratique. À vrai dire, lorsqu'on parle du mandat constitutionnel, la question qui est posée est celle de la durée au pouvoir, la question de la conservation du pouvoir.

C'est vrai que l'on est en droit de se demander si l'allongement de la durée des mandats présidentiels est un facteur d'appropriation personnelle du pouvoir. Nous pensons plutôt que c'est une remise en cause de la détermination du peuple congolais à créer un Etat de droit, de promouvoir le constitutionnalisme et de la démocratie populaire, beaucoup plus de sauvegarder la sécurité juridique et la souveraineté nationale appartenant au peuple.

Il est dit avec raison, que « la meilleure architecture constitutionnelle au monde dépend avant tout de la qualité d'hommes devant l'animer et d'un certain consensus que ce soit au niveau des élites ou de diverses composantes du peuple. Bien que l'optimisme soit de rigueur dans certains milieux, il ne semble pas encore évident qu'un tel consensus existe d'ores et déjà et que tous maîtrisent les termes du contrat qui comme tout autre contrat synallagmatique comporte des droits et des devoirs pour chacune des parties ».

Le constitutionnalisme et la démocratie ont plusieurs défis difficiles, du reste surmontables. Le peuple congolais doit comprendre qui ne lui sera pas servi sur un plateau d'or. La consolidation du constitutionnalisme et de la démocratie passe essentiellement par la protection des acquis de ceux-ci contenu dans la Loi fondamentale.

Somme toute, ni les concertations nationales, ni le dialogue inclusif, ni autre concept qu'on peut inventer pour réunir autour d'une même table une certaine couche de peuple congolais, ne saurait faire sauter les verrous de cet article. La seule révision acceptable dans ce cas, c'est la « révision-ajout» tendant à « intangibiliser » l'article 220 dans le souci de dissiper tout malentendu en se verrouillant expressis verbis comme tel est le cas de l'article 197 de la Constitution belge.

\section{BIBLIOGRAPHIE}

Akele Adau Pierre, Réponses pénales au discours du désordre ou au désordre du discours constitutionnel en République démocratique du Congo : la Cour constitutionnelle à l'épreuve, RADG, vol. 1, n ${ }^{\circ} 2$ et 3, 2014.

Beaud Olivier, La puissance de l'Etat, Paris, 1994.

Beaud Olivier, La souveraineté de l'Etat, le pouvoir constituant et le Traité de Maastricht : remarques sur la méconnaissance de la limitation de la révision constitutionnelle, Revue française de droit administratif, 1993.

Bolle Stéphane, Des Constitutions « made in » Afrique, CERDRADI, mai 2005.

Boshab Evariste, Entre révision de la constitution et l'inanition de la Nation, Bruxelles, 2013.

Boshab Evariste, Les dispositions constitutionnelles transitoires relatives à la Cour constitutionnelle de la République Démocratique du Congo, Fédéralisme Régionalisme, Volume 7, n¹, 2007.

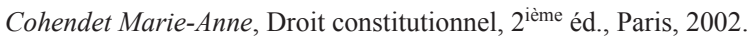

Delperee Francis, Droit constitutionnel, Tome 1, Bruxelles, 1987.

Duhamel Olivier, le quinquennat, Paris, 2000. 
Fall Ismailla Madior, Une démocratie « ancienne » en mal de réforme : Rapport sur l'état de la démocratie et de la participation politique au Sénégal, Johannesburg, 2012.

Formery Simon-Louis, La Constitution commentée. Article par article, 16e éd., Paris, 2013/2014.

Gauchet Marcel, La Révolution des pouvoirs, Paris, 1995.

Genevois Bruno, Les limites d'ordre juridique à l'intervention du pouvoir constituant, RFDA, vol.14, septembre-octobre 1998.

Hamon Lambert, La souveraineté nationale, la Constitution et les négociations 'européennes' en cours, 43 e Cahier, Chronique - LV, Recueil Dalloz Sirey, 1991.

Kazadi Mpiana Joseph, La révision constitutionnelle congolaise du 20 janvier 2011 : considérations critiques d'un citoyen (juriste). p. 12, http://www.droitcongolais.info (consulté le 15 décembre 2014).

Mbata B. Mangu André, Monarchies présidentielles et révisions constitutionnelles : le syndrome du troisième mandat ou d'une présidence à vie dans les Etats membres de l'union africaine, RADG, vol.i., n $1,2014$.

Renoux Thierry-Serge et M. Villiers, Code constitutionnel, Paris, Litec, 2005.

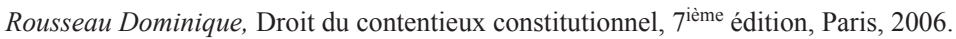

Rouvillois fréderic, Droit constitutionnel. 2. La Ve République, Paris, 2004.

Verdusen M. Stéphane, La procédure de révision de la Constitution: données comparatives, in Delperee Francis, et H. De croo, La procédure de révision de la Constitution, Bruxelles, 2003.

Verpeaux Michel, La Constitution, Paris, 2008.

Yatala Nsomwe Ntambwe Constantin, La révision constitutionnelle et l'intangibilité de l'article 220 de la Constitution congolaise, http://www.droitcongolais.info/files/REVISION-220-CSTRDC-II.pdf, (consulté le 12 décembre 2014). 